\title{
Prácticas, sujetos y conflictos en el campo de la justicia laboral brasileña a mediados del siglo $\mathrm{XX}$
}

Victoria Basualdo*

DROPPA, Alisson. Direitos trabalhistas: legislação, Justiça do Trabalho e trabalhadores no Rio Grande do Sul (1958-1964). Curitiba: Editora CRV, 2018.

Palabras clave: Justicia de Trabajo; Trabajadores; Rio Grande do Sul

Este libro tiene como principal objetivo analizar el funcionamiento de la Justicia de Trabajo en el estado de Rio Grande do Sul, en el período 1958-1964, un tiempo clave de movilización política de la clase trabajadora en Brasil. Está dividido en cuatro capítulos: en el primero se presentan los debates y controversias en torno de la organización y la ampliación de la Justicia del Trabajo en la década del 50 y los intentos de reglamentación del trámite jurídico de los procesos colectivos a través del poder normativo. El segundo capítulo aborda específicamente la evolución del derecho a la huelga, analizando específicamente la legislación regulatoria de las huelgas vigente entre 1946 y 1964. En el anexo del libro se ofrece adicionalmente una sistematización muy interesante de respaldo del análisis, mostrando los diversos proyectos y controversias en torno al derecho a huelga. El tercer capítulo analiza las diferencias de los procesos colectivos desarrollados en el marco de la Justicia del Trabajo, comparando diversas formas en que los sindicatos utilizaron este recurso judicial. El cuarto capítulo aborda procesos individuales, con el objetivo de analizar cuáles fueron procesados y cómo, lo que permitiría definir si efectivamente se logró una ampliación de los derechos de los trabajadores en el período comprendido entre 1958 y 1964. En conjunto, esta és la investigación desarrollada en el marco de la tesis en el Programa de Doutoramento em História Social da Cultura en el Instituto de Filosofia e Ciências Humanas - IFCH de la Universidade Estadual de Campinas - UNICAMP, Brasil.

El trabajo tiene una serie de méritos e impactos que resulta importante destacar. En primer lugar, ubica este proceso en una perspectiva histórica. Focalizándose en el período inmediatamente anterior al golpe de 1964, se remonta sin embargo al proceso de redemocratización a mediados de los años 40 con una presencia de una clase trabajadora con interés en la lucha y defensa de sus derechos, y en el marco de los años 50 a los que caracteriza, hasta el golpe de 1964 como una etapa muy intensa en la historia política de Brasil, caracterizada

* Doctora en Historia por la Universidad de Columbia, investigadora del CONICET y Área de Economía y Tecnología de FLACSO Argentina. 
por una ampliación de las huelgas y la participación de los trabajadores/as en diferentes ámbitos, incluyendo el poder judicial. Sostiene que el triunfo de Vargas en elecciones directas fortaleció el poder de los trabajadores implantado en los años 30 y consolidado en la década del 40, lo que evidencia, desde su perspectiva, la potencialidad de metamorfosis del sistema, y su capacidad de adaptarse a coyunturas muy distintas a aquellas originales del Estado Novo. Este trabajo profundiza en algunas de las formas de organización de la clase trabajadora para sostener la lucha por los aumentos salariales, la mejoría en las condiciones de trabajo y la ampliación de derechos, así como de las demandas en el marco de la Justicia del Trabajo permiten afirmar por un lado que el sistema tenía eficacia y por otro, que los trabajadores/as tenían fuerte interés en verlo funcionar. El libro muestra cómo estos fueron ingredientes importantes de un período muy singular, con huelgas, conquistas y también con significativa represión policial y en ocasiones, derrotas en los tribunales.

Ensegundolugar, yen vinculación conlo anterior, el librorealiza contribuciones importantes a la historiografía creciente sobre la dictadura cívico-militar en Brasil entre 1964 y 1985, así como sobre el período inmediatamente anterior. Leyendo esta investigación parece ineludible concluir que el funcionamiento de la estructura sindical, la evolución de la legislación social y las decisiones de la Justicia de Trabajo constituyeron factores centrales para tener en cuenta a la hora de explicar la reacción adversa y regresiva de las fuerzas armadas y del sector empresarial contra supuestas prerrogativas otorgadas por decisiones judiciales beneficiando a la clase trabajadora. Este trabajo se suma entonces a una creciente historiografía que enfatiza la importancia de considerar no sólo las dimensiones políticas de los procesos de radicalización política y social, sino también los aspectos de las relaciones laborales y el papel del movimiento sindical y la clase trabajadora, en particular para comprender procesos como el golpe de estado. Las reivindicaciones de la clase trabajadora gaúcha entre 1958 y 1964 no se habían logrado únicamente por una concesión del gobierno del estado, y no estaban vacías de contenido de clase. Los trabajadores también articularon otros temas que les eran esenciales, como la mejora de sus condiciones de vida, exigencias de aumentos salariales y la ampliación de derechos. En este sentido, el trabajo, aún cuando no aborda específicamente el período de la dictadura, sí marca en forma preliminar algunas tendencias interesantes. Señala como parte integrante del intento de reconfiguración de las relaciones laborales y las posibilidades de organización de los trabajadores tanto por vías represivas como institucionales, la limitación del poder normativo de la Justicia de Trabajo (por ejemplo en vinculación con la instalación de tablas de aumento salarial que en la práctica limitaron los derechos de negociación) así como los cambios regresivos en la aplicación de la ley de huelga analizada en el libro.

En tercer lugar, propone una mirada compleja del entramado institucional de los Tribunales del Trabajo. Sostiene que aunque la legislación social y la Justicia de Trabajo en sus abordajes oficiales tenían un discurso unificador, centrado principalmente en la CLT de 1943, al observar la práctica de los desarrollos de los procedimientos jurídicos (las reclamatorias, las sentencias, los acuerdos y los textos doctrinarios producidos por abogados, fiscales, jueces y ministros), se pone en evidencia la existencia de un campo de lucha, con frecuentes avances y retrocesos de los distintos campos y sujetos. El análisis detallado de cada uno de estos actores permite una aproximación muy interesante a las formas de interpretar el derecho a partir del estudio de decisiones tomadas por distintas instancias del 
poder judicial. Se logró en este trabajo reconstruir no una historia institucional descarnada, sino el análisis de lo que denomina"una institución viva", construida no sólo por los denominados "operadores de justicia" (ministros, desembargadores, jueces, abogados, fiscales, etc.), sino también por los empleadores, sindicatos y trabajadores/as que la utilizaron como un espacio de resolución de conflictos y de construcción de derechos. Esto permitió iluminar no sólo las relaciones de empleadores y trabajadores con el poder judicial, sino también los cambios en las percepciones de los jueces sobre la Justicia del Trabajo en temas clave, como el derecho de huelga.

En cuarto lugar, cabe detenerse en los aportes en términos de fuentes y metodología empleados en esta investigación, en la que se incluyó una amplia gama de fuentes. En primer lugar, se recurrió a documentos e información del Tribunal Regional de Trabajo de la Cuarta Región por medio de su Memorial y de la Coordinación de Gestión Documental del TST. Se realizaron además entrevistas que resultaron centrales para abordar temas en cada uno de los capítulos. Se consultaron además fuentes de la Facultad de Derecho de la Universidad de San Pablo y de la Universidad Federal de Rio Grande do Sul, del Instituto Brasileiro de Geografia e Estatística, la Fundação de Economia e Estatística de Rio Grande do Sul, la Fundação Escola da Magistratura do Trabalho de Rio Grande do Sul, la Associação dos Juízes Classistas de Rio Grande do Sul y el Museu Hipólito José da Costa.

En términos específicos, se concentró la investigación en 419 casos colectivos involucrando sindicatos de Porto Alegre, Rio Grande do Sul, en el período entre 1958 y 1964, los cuales permitieron analizar diversos aspectos de la lucha de los trabajadores/as y sus estrategias para obtener la efectivización de sus derechos. En la base de datos del Supremo Tribunal Federal (STF) se analizaron 233 acuerdos que tenían que ver principalmente con cuestiones relativas al derecho de huelga. También fueron estudiados 5401 procesos judiciales individuales iniciados por trabajadores/as en el marco de la Junta de Conciliación y Juzgamiento (JCJ) de Porto Alegre, lo cual permitió analizar las motivaciones que llevaban a trabajadores/as y patrones a recurrir a los tribunales. Al mismo tiempo, se consultaron diversos periódicos nacionales e internacionales para registrar menciones a debates o discusiones de legislación y decisiones judiciales, registrando aspectos doctrinarios relativos a los temas analizados. En términos metodológicos, la organización de toda la información en un formato de base de datos realizada en Excel permitió un cruzamiento y tabulación de los contenidos analizados a lo largo de todo el trabajo.

En quinto y último lugar, se explicitan y visibilizan en este libro campos de producción académica, equipos de trabajo y redes muy valiosas para la producción de conocimiento en forma compleja y sustantiva. Por un lado se destacan una serie de instituciones clave de UNICAMP, en cuyo marco se realizó la tesis doctoral, con la dirección de investigadores expertos que contribuyeron a consolidar un campo de estudios de gran interés sobre estos temas, y son clara demostración de esta presencia el prefacio y posfacio de los investigadores expertos Fernando Teixeira da Silva (Director de la Tesis Doctoral) y de Magda Barros Biavaschi. Por otro lado resulta muy importante también la vinculación con otros equipos, puesta de manifiesto en el libro en el prólogo de Angela de Castro Gomes, y la pertenencia y articulación con el colectivo Mundos do Trabalho, que permitió apuntalar y sostener miradas interdisciplinarias y de enorme riqueza. Pero además se ponen de manifiesto en este trabjaolas relaciones con equipos y proyectos de 
investigación de otros países de América Latina, para los cuales esta investigación también reviste enorme interés.

En base a todas estas fortalezas, este libro logra un valioso análisis del funcionamiento del poder normativo de la Justicia de Trabajo por medio de los casos colectivos iniciados en el TRT4, entre 1958 y 1964, por los sindicatos de empleados y patrones en la ciudad de Porto Alegre en Rio Grande do Sul, sumando este análisis de caso regional a un campo muy rico y fértil de estudios sobre distintas localidades y territorios del Brasil, y permitiendo avanzar hacia una mirada nacional, con impactos y potencialidades para la historiografía de la región. A lo largo de los capítulos puede verse cómo la lucha por los derechos articuló no sólo los debates y discusiones en torno a la legislación referida al derecho a huelga, sino también las batallas cotidianas llevadas adelante tanto por trabajadores como por patrones, el movimiento sindical, la estructura del estado y las formas de organización en el lugar de trabajo. Por todo esto, el presente libro constituye entonces una relevante y significativa contribución a la comprensión de la experiencia de la clase trabajadora brasileña en el campo del derecho en una etapa clave de su historia.

Recebido em 21/12/2018

Aprovado em 21/12/2018 